Meta

Journal des traducteurs

Translators' Journal

\title{
Contextual Transposition in Translating Research Instruments
}

\section{Yvon Gasse}

Volume 18, numéro 3, septembre 1973

URI : https://id.erudit.org/iderudit/002810ar

DOI : https://doi.org/10.7202/002810ar

Aller au sommaire du numéro

Éditeur(s)

Les Presses de l'Université de Montréal

ISSN

0026-0452 (imprimé)

1492-1421 (numérique)

Découvrir la revue

Citer cet article

Gasse, Y. (1973). Contextual Transposition in Translating Research Instruments. Meta, 18(3), 295-307. https://doi.org/10.7202/002810ar d'utilisation que vous pouvez consulter en ligne.

https://apropos.erudit.org/fr/usagers/politique-dutilisation/ 


\section{Contextual transposition in translating research instruments}

\section{INTRODUCTION}

In this paper, we are mainly interested in the problem of translation in general and particularly in the translation of research instruments for the purpose of crosscultural studies. This interest was developed from a personal experience when faced with the difficulties of using questionnaires in a bilingual country, in this case, the Province of Quebec.

In the first part of the paper, we have attempted to review the modern nature of translation after trying to answer some fundamental questions about this process. Having accepted the fact that translation is closely related to language per se, we have made a brief incursion in the linguistic science in order to assess the position of translation within it. A theory of translation can be derived from this linguistic science and its elaboration is rapidly illustrated.

In the second part, we have tried to elaborate the main argumentation of this paper, that is, the relation between context, bilingualism, and translation. The discussion revolves around the importance of linguistic, cultural, and situational contexts as major determinants of a precise translation. From this, a modified theory of translation has emerged which we have called « contextual transposition 》.

The last part is devoted to studying the application of this \& theory $>$ in the translation of research instruments in cross-cultural studies and research made in a bilingual country with special references to the Province of Quebec.

Nature of translation

There are two trends in contemporary translation : traditional translation depending on the competence of a person and his knowledge of two languages; 
and machine translation depending of computers. Even if many studies have been conducted on machine translation, especially since the last ten years, we are not concerned with it in this paper; instead we will restrict ourselves with the most up to date studies of the traditional translation.

Developments in linguistic theory have shed important light upon the theory and practice of translation, resulting in the recognition that translating is basically not a process of matching surface forms by rules of correspondence, but rather a more complex procedure involving analysis, transfer, and restructuring. Such linguistic procedures as transformation, and componential analysis and such special techniques as decentering and back-translation provide for more satisfactory bases for translation than have existed in the past.

At the same time, the theory of translation is able to provide linguistic science with new insights into structure and with improved methods for testing hypotheses.

A review of the literature on this subject shows a certain level of discrepancy on a precise definition of the translating process. Of course, there is a fair amount of agreement on the very basic function of translation (i.e., linguistic transfer from a source language to a target language) but variances occur between translators according to their perspectives on translation scope and accuracy.

Nida ${ }^{1}$ provides us with a definition which seems a good reflection of modern translation when he sees the process as reproducing in the receptor language the closest natural equivalent of the message of the source language, first in terms of meaning and second in terms of style. Implicit in this definition is the reproduction of context which determines in fact the meaning.

Another difficulty lies in the ever present question : Is translation a science or an art? It could be a science, a skill or an art depending on the particular functional level from which it operates. If the translator is seen as a creative artist, and above all an indissoluble and inseparable alter ego of each writer, poet, and thinker then translation is an art. On the other hand, if we consider a theory of translation as a special domain of linguistics (comparative or applied linguistics) because its' basic role lies in language, then we can speak about a science. On this point, translation can be considered as a second degree linguistic creation. It cannot be separated from linguistics; it deals with problems of relationships between thought and language; problems of the role of language in the process of knowledge; problems of correlations between linguistic systems and types of national mentality. Finally, if we look at the requirements to constitute a good translator, we may certainly say that translation is a skill. The translator must have a perfect knowledge of the language of the author whose work he translates, and he must be similarly expert in the language into which he performs the translation. Also he must understand and be able to reason about the subject matter to be translated.

Viewing translation from those three functional levels we are led to wonder if translation is even possible after all. In an absolute sense, we may say that it is 1. Eugene A. Nida, « Science of Translation ", Language, XLV, 3 (1969), p. 495. 
impossible ; in this case we are talking about a perfect translation, but in practice we are more interested in various levels of adequate translation.

This idea of the impossibility of translation is based on different linguistic conceptions elaborated by W. von Humboldt in his classical book $\ddot{U} b e r$ die Kawisprache (1836-1840) in which he argued that each language is like a big circular fence around people who talk this language and if we go over this fence then we fall in another circular boundary; for him, people's language, it is their spirit and people's spirit it is their language. Then according to Humboldt each language represents a finite system which expresses people's mentality and it is impossible to translate this mentality by the particular means of another mentality.

Humboldt's ideas have been developed by many other linguists who have drawn extreme conclusions. Among those is the famous "Sapir-Worf hypothesis » on linguistic relativity.

Sapir ${ }^{2}$ described how perfectly language and culture mesh. Different cultures analyze the world differently, according to what experiences are important to them. People contact the environment through the medium of their culture's language, which emphasizes some details and omits others. Language reflects how a culture views the world, thereby guiding its users to social reality.

Whorf ${ }^{3}$ hypothesizes from these observations and carries them a step further : the way men think is determined by the language system of their culture. In fact, they are locked into cultural ways of perceiving and knowing the world. Whorf attempted to prove that the language shapes thought by the method of literal translation. Any translator knows that literal translation never really gives the intended sense of what is said or written. It is impossible, therefore, to prove on the basis of literal translation, that people carry totally different worlds in their minds.

In a cross-cultural study conducted by $\mathrm{L}$. A. Jakobovits ${ }^{4}$ to test the Whorf hypothesis, it is demonstrated that this hypothesis ignores many important facts and it is contradicted by the evidence for generality presented here and elsewhere.

Rather than being impressed by the impossibilities of translation, as Nida ${ }^{5}$ pointed out, anyone who is involved in the realities of translation in a broad range of languages is impressed that effective interlingual communication is always possible, despite seemingly enormous differences in linguistic structures and cultural features.

\section{Linguistics and translation}

The structuralist approach, one of the great trends in social anthropology and sociology have been extensively applied in linguistic theories. To a considerable

2. E. Sapir, "The Status of Linguistics as a Science ", in D. G. Mandelbaum, edit., Culture, Language and Personality, Berkeley (Calif.) University of California Press, 1958.

3. B. L. Whorf, "Science and Linguistic », Technology Review, XLIV (1940).

4. L. A. Jakobovits, "Comparative Psycholinguistics in the Study of Culture", International Journal of Psychology, I, 1 (1966).

5. "Science of Translation", Language, XLV, 3 (1969), p. 483. 
degree, Noam Chomsky ${ }^{6}$ accomplished the eclipse of this behaviorist approach to language with his notions of transformational grammar. It is based on the hypothesis that all human languages share deep-seated properties of organization and structure.

The simple aspect of transformational grammar analyzes language in terms of surface and deep structures. Surface structure carries sounds and vocabulary of a language; it shows the organization of the sentence into categories and phrases. The underlying deep structure is also a system of categories and phrases, but with a more abstract character ; it extracts from the complex idea that constitutes the subject of the surface structure an underlying proposition; it is the deep structure which expresses the meaning of the sentence.

According to Chomsky ${ }^{7}$ the knowledge of a language involves the ability to assign deep and surface structures to an infinite range of sentences, to relate these structures appropriately, to assign a phonetic interpretation to the surface structure and to construct a semantic interpretation on the basis of the grammatical relations of the deep structure. If this approach is correct, then a person who knows a specific language has control of a grammar that generates or characterizes (generative grammar) the infinite set of potential deep structures and maps them onto associated surface structures.

According to this transformational grammar, the structural differences are much more significant at the surface level than at the deep structural level. Thus, deep structures could be considered as universals. This process has been very well illustrated by Werner and Campbel1 ${ }^{8}$ in a scheme of a Unified Theory of Language.

From this aspect, translation could be defined as a text analysis of language A in terms of deep structures and the conversion or transfer of the latter, through transformation, into surface structures of language $B$.

$\mathrm{Nida}^{9}$ suggests a similar method of translation but with a major exception : instead of making the transfer at the deep structure level we must make it at a level higher, that is, at the kernel or core structure level.

\section{Theory of translation}

Many procedures and theories of translation have been suggested in the last ten years but they are all more or less technical and mechanical. However, a group of those theories seem to be more sophisticated and more complete and they are based on similar properties as described by Chomsky and Nida. They could be represented diagrammatically in the following figure :

6. N. Chomsky, «Language and the Mind », Psychology Today, I, 9 (1968).

7. N. Chomsky, "Problems of Explanation in Linguistics », in Robert Borger and Frank Cioffi, edit., Explanation in the Behavioral Sciences, Cambridge, Cambridge University Press, 1970, p. 430.

8. Oswald Werner and Donald T. Campbell, \& Translating, Working through Interpreters, and the Problem of Decentering ", in R. Naroll and R. Cohen, edit., $A$ Handbook of Method in Cultural Anthropology, Garden City (N.Y.), The Natural History Press, 1970, p. 401 .

9. "Science of Translation», Language, XLV, 3 (1969). 


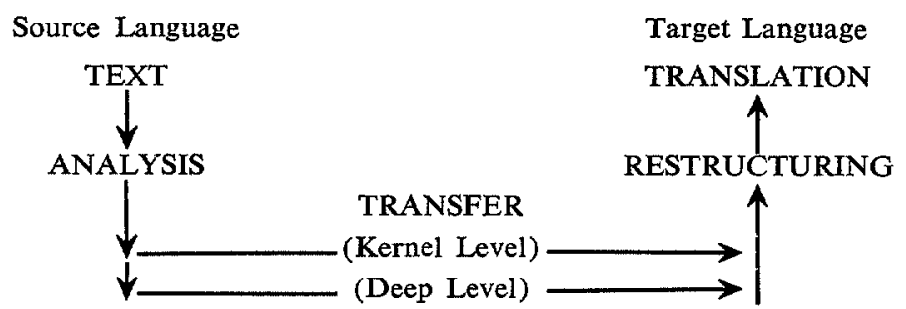

As we said earlier, the transfer can be made at different levels. If the translation is done at the surface level, then we can speak about a literal or word-forword translation; the equivalence is established at the lowest rank of the hierarchy of translation. The unit of equivalence is the word without much concern for the meaning and style. This kind of translation is not very used because it is not accurate.

Nida ${ }^{10}$ has called that, a translation oriented toward formal-equivalence. Such a principle may be pushed to an absurb extent, with the result being relatively meaningless strings of words. A consistent literal translation will obviously contain much that is not readily intelligible to the average reader. Some types of strictly surface translation, e.g., interlinear renderings and completely concordant translations are of limited value; others are more interesting for certain specific purposes.

When the transfer is made at the deep structure level, we encounter problems which are at the opposite extreme of the continuum. These difficulties are related to the principle dealing with the level of abstraction. The higher the level of abstraction, the more general is the meaning of a statement.

Again, the deep structure level is that of language universals and on this respect the meaning in different languages tends to be similar. As Nida ${ }^{11}$ pointed out the deepest structural level involves simply a pool of semantic universals. On an absolute basis, translation at this level should be the « ideal $\gg$ because meaning is supposed to be the most equivalent at the most abstract degree; but at the same time we loose some parts of the context itself and the equivalence in meaning is then restricted to the words or units out of context and there is a greater risk of skewing. In other words, we cannot manipulate the structure within the context at this level of abstraction.

Another problem arises when it is time to transform toward the deepest structure; the more general and similar the meaning of a unit the more difficult and complex it is to reach. So, here as elsewhere we have to compare the results versuls the efforts and the law of diminishing returns applies very rapidly in this case.

In order to overcome those difficulties, $\mathrm{Nida}{ }^{12}$ proposed to transfer at the core or kernel level. There are many advantages to work at this level. The translator can set up structural correspondences which he can readily manipulate in

10. Eugene A. Nida, Toward a Science of Translating, Leiden, E. J. Brill, 1964, p. 165.

11. Ibid.

12. Ibid. 
his mind. The analysis of the text provides a series of kernels or core sentences, so it is easier to fit the right one to the relevant context and then establish a correspondence with a kernel in the target language. The translator must have these kernels related meaningfully to one another. This means that he must back up from a strictly kernel level and analyze the relationships between kernels. Compared with the deep structure level, the kernel level requires less work and it is easier to find out relationships and meanings which are more practical and relevant.

Translation at this level has been called by Nida 13 \& translation oriented toward dynamic equivalence $\gg$. It is the closest natural equivalent to the sourcelanguage message. The important words here is «natural equivalent $;$; this means an almost complete adaptation to the receptor language context. The important features of this translation lie in the fact that it is not a literal translation and it can be manipulated in order to fit the right context in the target language. And agreeing with Nida ${ }^{14}$, it is important to realize that a dynamic equivalence translation is not merely another message which is more or less similar to that of the source. It is a translation, and as such must clearly reflect the meaning and intent of the source.

End steps of the whole process are characterized as analysis and restructuring. Analysis consists in the decomposition of a whole sentence in its major components; and making different associations between those particular units or some external components closely related (kernel or core sentences). In this way, it is possible to evaluate the different semantic outputs and then choose the most appropriate one according to the context.

While for Nida ${ }^{15}$ analysis is operated by back-transformation, for Werner and Campbell ${ }^{16}$ it is by decentering with the means of back-translation. They are very important processes even if they have been almost completely neglected in the past in translation; the implicit principle stated that we do not change the source text. In reality, back-transformation or decentering is not a change in the text but a clarification.

The restructuring step is certainly the most difficult operation in translation. It consists essentially in building from a certain number of kernel sentences in the receptor language, within the new cultural context, a whole sentence which will be the natural equivalent of the same sentence in the source language. At this point, the problem is one of contextual comparison between the source and target cultures.

\section{2}

\section{Context and translation}

The problem of context seems to me one of the most important in translation because people can and do interpret words in entirely different ways depending upon the situation. To understand the meaning of a word, we must know, in addition

13. Toward a Science of Translating, p. 166.

14. Ibid.

15. "Science of Translation ", Language, XLV, 3 (1969).

16. "Translating, Working through Interpreters, and the Problem of Decentering ", in $R$. Naroll and R. Cohen, edit., op. cit. 
to the individual's experience with it, the present context (explicit and implicit) in which the word is used.

Words do not, of course, stand alone. In most languages, they are combined to form phrases, sentences, or larger « utterance units». We are concerned here with the explicit context as determined by the syntactical arrangement. Every word occurs in a context of other words, and the meaning of a word depends upon the pattern of these other words. And the meaning of the individual words in the sentences changes when the words are ordered in different ways.

At the kernel level, it is possible to manipulate the words in order to get the best explicit contextual pattern in the receptor language. This elementary linguistic pattern is the basis for a more elaborate one in the final translation. If an explicit linguistic context is not supplied, the individual in the target language will provide his own internal verbal context with, of course, his own meaning.

In addition to this target linguistic context, the translator must take into consideration the implicit cultural and situational context of the receptor language. This activity occurs at the restructuring step of the final translation. We draw a clear distinction between the explicit linguistic context and the implicit cultural and situational context. For instance, you can take the same French utterance (same syntactical arrangement) in France and in Quebec and they have not the same meaning in both cultures.

The significance of context has been well pointed out by Church ${ }^{17}$ when he suggested that words do not have meanings, but functions. The «meanings 》 assigned to words by dictionaries are abstractions drawn from the ways words function in various contexts.

For Slama-Cazacu ${ }^{18}$ the role of context consists in choosing the right words and in precising among the multiple meanings (polysemy) the most appropriate ones. In this sense, then the context individualizes, completes and even creates the meaning of a word or of a sentence.

This problem of context is closely related to the amount of information in a given situation. The more context we have, the less information we need and vice versa. This case happens frequently in the translation of research instruments ; the amount of information is usually low, then we must provide more context.

Werner and Campbell ${ }^{19}$ suggest that all methods of decentering translation are interpretable as methods for providing more context in both languages. Backtranslation is a special case for providing more context by mapping equivalent sets of sentences.

In a study on "Items in Context , Longacre ${ }^{20}$ suggested that translation is most successful in transmitting the message of a text as a whole and less successful in reproducing details of immediate context.

Bilingualism is a case of special context in translation.

17. J. Church, Language and the Discovery of Reality, New York, Random House, 1961.

18. Tatiana Slama-Cazacu, Langage et contexte, The Hague, Mouton \& Co., 1961, p. 210.

19. "Translating, Working through Interpreters, and the Problem of Decentering ", in R. Naroll and R. Cohen, edit., op. cit.

20. Robert E. Longacre, "Items in Context », Language, XXXIV, 4 (1958), p. 491. 


\section{Bilingualism and translation}

Bilingualism is important in translation in many ways. There are different degrees of bilingualism and many ways to acquire different languages. The translator is quite affected by these characteristics and must be aware of them in order to get his translation as symmetrical as possible. If the translation is done on research instruments and the sample is made up of bilingual people (e.g., Province of Quebec), then bilingualism is of great importance.

Usually, two kinds of bilingualism are identified; these distinctions have been made by Osgood and Sebeok ${ }^{21}$. The compound bilingual has one set of representational processes which may be associated with either of two sets of linguistic responses. Compound bilinguals are frequently produced in foreign language classrooms where a second language is taught by linking it with translations in the native language. Here, the supposition is that each language draws on the same system of meaning or in our terminology on the same cultural context.

On the other hand, coordinate bilinguals have two sets of representational processes which are not identified but parallel. Each mediation is associated with an appropriate set of linguistic responses. Usually, persons with coordinate bilingualism have learned the two languages in two different cultural contexts.

This distinction is not a dichotomy; bilinguals can be distributed along a continuum from a pure compound system to a pure coordinate system.

The big problem encountered in translation in a bilingual context is that of interference. Or course, interference occurs more often in a compound bilingualism but even within a coordinate system there may be interference between the two languages. Interference is most likely to occur when the languages are closely related and the cultures or the experiences associated with the languages are alike (e.g., English and French in the Province of Quebec).

This assertion has been proved by Lambert et al. ${ }^{22}$ when they found that experience in separated contexts (coordinate bilingualism) comparatively increases the associative independence and semantic differences of translated equivalents in the bilingual's two languages (in this case, English and French). Interference is that kind of constant pressure on the bilingual to confuse meanings, to interpret a sign in language $\mathrm{A}$ as its translation-equivalent in language $\mathrm{B}$ would be interpreted.

This process of interference is acting differently depending upon whether the translator maintains compound or coordinate languages in his nervous system, and, if the former, whether he is translating to or from his dominant language.

In the case of the compound bilingual, more difficulties will occur when he will translate from his dominant language because his familiar context will be

21. E. E. Osgood and T. A. Sebeok, edit., "Psycholinguistics : A Survey of Theory and Research Problems ", A Morton Prince Memorial Supplement to The Journal of Abnormal and Social Psychology, XLIX (1954), p. 139.

22. W. E. Lambert, J. Havelka and C. Crosby, "The Influence of Language-Acquisition Contexts on Bilingualism ", The Journal of Abnormal and Social Psychology, LVI, 2 (March 1958), p. 239-245. 
confused with the target cultural context. If he translates to his dominant language, then he may misinterpret the source cultural context.

On the other hand, the coordinate bilingual faces very different difficulties. Because he knows both languages and cultures « equally (for our purpose), he has the tendency to jump over the two mediation steps, i.e., analysis and restructuring. In other words, he translates at the near surface level loosing the benefits of the transfer at the kernel level.

More interference will occur if the people to whom the translation is intended are themselves compound bilinguals. Usually, even if one language is dominant, they do not master it very well as monolingual would do. So, they are often confused in both languages and in reality they are talking a third language derived from their bilingualism. In this case the right context is difficult to reach.

But as many studies done by Lambert ${ }^{23}$ and by Davis and Wertheimer ${ }^{24}$ have demonstrated, a linguistic dominance seems to exist on all bilinguals (in this case, English and French). This dominant language is usually the mother tongue. Even for the coordinate bilinguals, there is a dominant language. Then translation can be worked out on the basis of the dominant language but not in a like-monolingual way.

\section{Contextual transposition}

After reviewing what general translation and bilingualism are and what we meant by context we characterize a "precise » translation as a contextual transposition. Transposition, in the sense we choose to give it, occurs when the language of the matter to be translated stands close enough to the language of the translator, in age, idiom, cultural habits and so on, for him to be able to follow the letter (linguistic context) with a fair hope of keeping faith with the spirit (cultural context).

Turning a modern French novel into English is thus mainly a matter of transposition. But as Carne-Ross ${ }^{25}$ pointed out, the further one moves back in time, the more transposition must approximate pure translation. He thinks that one should transpose when one can; and only translate when one must.

Our argumentation here is based on the fact that it is almost impossible to translate a context; however we can transpose it, i.e., from a source frame of reference to a target frame of reference. The process is similar to that described earlier except that we distinguish two different transfers or contextual transpositions.

23. Wallace E. Lambert, a Measurement of the Linguistic Dominance of Bilinguals \#, Journal of Abnormal and Social Psychology, L, 2 (March 1955), p. 197-200; Wallace E. Lambert, « Developmental Aspects of Second-Language Acquisition : I. Associational Fluency, Stimulus Provocativeness, and Word-Order Influence », The Journal of Social Psychology, XLIII (1956), p. 83-89.

24. Bonnie J. Davis and Michael Wertheimer, "Some Determinants of Associations to French and English Words », Journal of Verbal Learning and Verbal Behavior, VI (1967),

25. D. S. Carne-Ross, Translation and Transposition ", in William Arrowsmith and Roger Shattuck, edit., The Craft and Context of Translation, Austin, the University of Texas Press, 1961. 


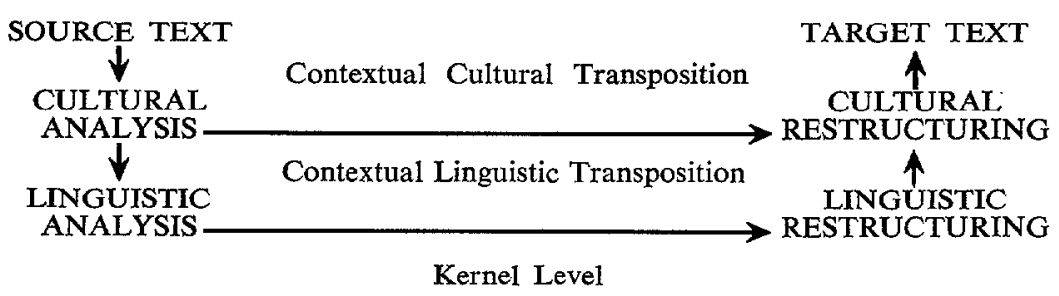

The linguistic analysis is done within a very rigid and formal structure, i.e., syntactic, grammatical semotactic, etc., which is peculiar to a language (e.g., while in English the adjective is usually placed before the noun, in French it is placed after.) In this process, the translator has no liberties; he must choose the best translation-equivalent following the linguistic rules. This contextual linguistic transposition is in most cases not sufficient because it does not take into consideration culture and the situation.

It is then the role of our second transposition to provide for this incompetence. In this contextual cultural transposition (culture and situation) the translator must adjust and fit his target linguistic restructuring to the particular culture and situation to which the translation is intended. Back-transformation between the two restructurings is very helpful here.

It is this cultural non-fit which accounts for the difficulty to understand many French texts, written or translated in France, when they are used in Quebec or vice versa. It is also this situational non-fit which accounts for many incomprehensibilities of translated scientific and technical texts. In both cases the linguistic context may be similar but not the cultural and situational context.

I think it is this idea which led Edmond Cary ${ }^{26}$ to argue that translation is not a linguistic process (he should say : not only a linguistic process) but a process involving facts bounded to a whole cultural context (he should say : a process involving linguistic facts as well as a cultural context).

We must notice here that our process works best when the translator knows the situation or the culture of the target language and when he has a fair amount of experience in both of them. It is in this perspective that we view the translation of research instruments.

\section{Translation of research instruments}

In cross-cultural research and in research in a bilingual country it is necessary to translate the research instruments (questionnaires, attitude scales, interview schedules, special techniques, instructions, etc.) and on the accuracy of this translation will depend to a certain extent the validity and reliability of the results.

As Campbell et al. ${ }^{27}$ showed, it is quite surprising to note that this problem of translation accuracy has not been more taken into consideration as an important

26. Edmond Cary, la Traduction dans le monde moderne, Genève, Georg et Cie, 1956.

27. D. Campbell, R. Brislin, V. Stewart and O. Werner, "Back-Translation and Other Translation Techniques in Cross-Cultural Research", paper to be submitted to the International Journal of Psychology (1970). 
parameter for data distortion in cross-cultural studies. And as Richard Crosby ${ }^{28}$ pointed out, these is a shocking dearth of specific research bearing on the whole problem of cross-cultural attitude measurement.

Translation of research instruments seems a good domain for the application of our process of contextual transposition. People to whom the translation is intended are usually known and the situation well defined so the context is more readily accessible to the translator.

Two cases can occur in this kind of research : a) Instruments used are standard or have been designed by somebody external to the research team. $b$ ) Instruments are designed by the research team and specifically for this particular research.

In both cases, the ideal situation is when the translator is himself a member of the research team; then he is already acquainted with the object of the research, the situation, the sample and its methodology; in other words, he knows the source context. Within this ideal situation, the best is when the translator transposes into his dominant language.

Because the research instruments are usually made up of short paragraphs, single sentences, utterances, idioms, special expressions or even single words, the amount of information is very low and it becomes necessary to provide more context in order to get the exact meaning.

When the instruments are standard, it is more difficult to change the questions or items of the source if we want to compare with past results; in this case the cultural context transposition is the most important one. We must find out the same item, having the same meaning in the same situation in the receptor language. If it is impossible to get a similar expression, then we must create one in building the entire context (explanation or illustration).

When the instruments are designed by the research team, the translator must be involved directly in its structure. In this case, we talk about the creation of the same instruments in two languages or more. In fact, there is no translation or transposition in the sense described earlier but instead a simultaneous creation from the same frame of reference. The items are chosen only if they have equivalence in both contexts.

On the other hand, if it is impossible to have the translator directly involved in the construction of the instruments, then transposition is necessary but here it is always possible to apply the back-translation techniques as described by Campbell et al..$^{29}$, for decentering the source language.

So far, we have assumed that the sample in both source and target languages was monolingual. In the case of a bilingual sample (different degrees of bilingualism) it is extremely important to have the items in both versions printed together on the same instrument; so people can answer the one they understand best. If they do not understand a certain expression in one language they will immediately check with the translation and usually, with both contexts, they get the right meaning. It is a simple way to provide for more context.

28. Richard W. Crosby, "Attitude Measurement in a Bilingual Culture », Journal of Marketing Research, VI (November 1969), p. 421-426.

29. " Back-Translation and Other Translation Techniques in Cross-Cultural Research», paper to be submitted to the International Journal of Psychology (1970). 
This assertion is based on a personal experience when doing exploratory research in the Province of Quebec's business enterprise. We have used bilingual questionnaires because people did not understand very well in just one language.

The reason for this is very simple; bilingual people learn and use languages differently. In Quebec for example, most people use a french syntactic and grammatical structure but with a French-English vocabulary (e.g., all business, commercial, technical and craft terms are learned and used mostly in English). This gives a third language called franglais; instead of using this, it is more appropriate to use both languages.

The only problem with this technique occurs when there is a real discrepancy between the same expression in both 1anguages. In this case people can be confused and they do not know which one to choose. Usually, people choose the one which is the easiest to answer or the one in their dominant language.

It is certainly important to pre-test or validate the questionnaires before using them in the field. Among the different validation methods available, we agree with Prince and Mombour ${ }^{30}$ that bilingual subjects should be used. We would like to add another possibility to the methods they suggested.

The bilingual instrument could be administered to the same persons asking them to answer according to their feelings in the language of the question or items. But items should be placed at random and not together as used in the field. In this way discrepancies between expressions judged equivalent a priori can be identified right away.

Here, like for any other kind of instruments, a multi-method validity and reliability test is necessary as suggested by Campbell and Fiske ${ }^{31}$.

\section{CONCLUSION}

After doing this brief analysis on the translation process, we are in a better position to evaluate the problems which may occur in cross-cultural research or in research in a bilingual setting. We are particularly interested to apply this contextual transposition in the Province of Quebec. The kind of bilingualism we have here is hard to evaluate because many words are learned directly in different contexts and many others by translation; but we would say that people are more compound bilingual than coordinate bilingual. So, in this case we see a real necessity to use both languages together in research instruments in order to eliminate all confusion.

The choice of a translator is also important; as we said earlier the kind of French spoken in Quebec is quite different from the French spoken in France; we would suspect the same thing (at a lesser extent) about the kind of English

30. R. Prince and W. Mombour, \&A Technique for Improving Linguistic Equivalence in Cross-Cultural Surveys », International Journal of Social Psychiatry, XIII (1967), p. 229-237.

31. D. T. Campbell and D. W. Fiske, «Convergent and Discriminant Validation by the Multitrait-Multimethod Matrix », Psychological Bulletin, LVI, 2 (1959), p. 81-105. 
spoken in Quebec. Only a Quebec native translator can find out his way in this labyrinth. This is so true that recently some Quebec French films have had to be «translated 》 in France French in order to be used in France.

Then, the fact that the translator is linguistically skillful in French and English is not sufficient; he must also have a fair amount of experience in both cultural and situational contexts of the Province of Quebec.

From the assessment of this particular situation, we do not know exactly to what extent it is possible to draw generalizations which could be applicable to other bilingual countries. A certainly plausible generalization is the fact that the contextual and situational analysis is not an extra in translation and especially in translation of research instruments but it is a must which one cannot avoid if one wants a «precise » translation.

YVON GASSE 